\title{
Síndrome cardiorrenal
}

\author{
Javier Eliécer Pereira-Rodríguez*, Lorena Boada-Morales, \\ Damaris Rocío Niño-Serrato, Myriam Caballero-Chavarro, \\ Gina Rincón-Gonzales, Tania Jaimes-Martín y Jésica Melo-Ascanio
}

\author{
Grupo Rehabilitar Cl, Colciencias, Cúcuta, Colombia
}

Recibido el 15 de agosto de 2016; aceptado el 19 de mayo de 2017

Disponible en Internet el 14 de noviembre de 2017

\section{PALABRAS CLAVE \\ Falla renal; Falla cardiaca; Remodelamiento ventricular}

\section{KEYWORDS}

Renal failure;

Heart failure;

Ventricular

remodelling

\begin{abstract}
Resumen La insuficiencia cardiaca origina inicialmente una lesión miocárdica que conlleva remodelamiento ventricular, lo cual induce a la activación de mecanismos compensadores, entre los cuales el riñón es pieza fundamental ya que regula la homeostasis hidroelectrolítica y así el volumen circulante. El sistema nervioso simpático y el sistema renina-angiotensinaaldosterona aportan una retención de sodio y agua que afecta negativamente la función cardiaca y conduce a compromiso cardiovascular, miocárdico y renal; de allí nace la definición clínica de síndrome cardiorrenal que se clasifica de acuerdo con su forma de presentación y componentes fisiopatológicos. Esto motivó la definición y conceptualización del síndrome cardiorrenal, que incluye interacciones bidireccionales, en la que alteraciones, tanto agudas como crónicas de cualquier órgano, pueden afectar de manera funcional o estructural la función ventricular, la renal o ambas.

(C) 2017 Sociedad Colombiana de Cardiología y Cirugía Cardiovascular. Publicado por Elsevier España, S.L.U. Este es un artículo Open Access bajo la licencia CC BY-NC-ND (http:// creativecommons.org/licenses/by-nc-nd/4.0/).
\end{abstract}

\section{Cardiorenal syndrome}

Abstract Heart failure initially causes myocardial damage that leads to ventricular remodelling. This, in turn, leads to activation of compensatory mechanisms where the kidney plays a fundamental role, as it regulates electrolyte homeostasis and thus the circulating volume. The sympathetic nervous system and the renin angiotensin-aldosterone system lead to the retention of sodium and water, which adversely affects cardiac function. This leads to cardiovascular, renal and myocardial compromise, or a cardiorenal syndrome, which is classified according to

\footnotetext{
* Autor para correspondencia.

Correo electrónico: rehabilitarcucuta.investiga@gmail.com (J.E. Pereira-Rodríguez).
} 
its presentation and pathophysiological components. The definition and conceptualization of cardiorenal syndrome includes two-way interactions, where acute and chronic changes of any organ can functionally or structurally affect the ventricular and/or renal function.

(c) 2017 Sociedad Colombiana de Cardiología y Cirugía Cardiovascular. Published by Elsevier España, S.L.U. This is an open access article under the CC BY-NC-ND license (http:// creativecommons.org/licenses/by-nc-nd/4.0/).

\section{Definición}

El síndrome cardiorrenal, también conocido como "síndrome de anemia cardiorrenal" $(\mathrm{SACR})^{1}$, es un estado de desregulación avanzada entre el corazón y el riñón ${ }^{2}$, que involucra la afectación de ambos órganos sistémicos ${ }^{3}$ debido a una disfunción aguda o crónica de uno de los órganos que induce a la disfunción del otro ${ }^{4}$, para lo cual se verán alteradas sus funciones fisiológicas en la relación corazón-riñón donde cualquiera de los dos órganos utilizará un mecanismo de compensación que tendrá una repercusión importante en el otro órgano ${ }^{5}$. Esta definición posee tres características fundamentales para entender este síndrome; la primera es una relevancia igualitaria entre ambos órganos, ya que muchas veces no queda claro donde inició el daño; la segunda, es que esta disfunción puede ser aguda o crónica y también funcional o estructural, y la tercera recalca el hecho de que la interacción es bidireccional ${ }^{1}$, características que conducen a un círculo vicioso negativo que se traduce en la descompensación de todo el sistema circulatorio ${ }^{6}$.

Existe una estrecha comunicación entre el corazón y los riñones a través de varias vías que incluyen la presión de perfusión renal, la presión de llenado cardiaco y la actividad neurohormonal, en particular del sistema nervioso simpático, del sistema renina-angiotensina-aldosterona y de los péptidos natriuréticos ${ }^{7}$.

La disfunción cardíaca asociada al empeoramiento hemodinámico ocasiona la activación de mecanismos compensadores en los órganos sistémicos, en cuyo caso el riñón desempeña un papel central ya que regula la homeostasis de electrolitos y volumen ${ }^{8}$ para lo cual los trastornos del corazón y los riñones inducen la disfunción aguda o crónica del otro, influenciando en los efectos negativos sobre los cardiomiocitos, el endotelio, los hematíes y el complejo glomerular. Así mismo, la lesión que desencadena la activación del sistema renina-angiotensina-aldosterona, péptidos natriuréticos y vasopresina se ve exponencialmente exacerbada por el arsenal proinflamatorio de citoquinas que condenan al fracaso a los insuficientes mecanismos compensadores ${ }^{9}$.

Ahora bien, tanto el corazón como los riñones son ricamente vascularizados (los riñones son más vasculares que el corazón) y ambos tienen inervación simpática y parasimpática.

Estos dos órganos actúan en conjunto regulando la presión arterial, el tono vascular, la diuresis, la natriuresis, la homeostasis del volumen intravascular, la perfusión tisular periférica y la oxigenación ${ }^{10}$. También tienen funciones endocrinas con acciones hormonales fisiológicas interdependientes reguladas por el péptido natriurético arterial, un vasodilatador secretado por el sistema reninaangiotensina-aldosterona $(\mathrm{SRAA})^{10}$. Al mismo tiempo, el síndrome cardiorrenal se presenta en pacientes que se encuentran en una situación clínica en la que hay compromiso simultáneo de la función cardiaca y renal ${ }^{9}$. La relación entre las dos enfermedades es amplia y no resulta fácil establecer límites sobre lo que pertenece a cada una; entre un 30 a un $50 \%$ de los pacientes con insuficiencia cardiaca padecen enfermedad renal crónica y esto representa un factor de mal pronóstico ${ }^{6}$. Por otra parte, es importante conocer que la insuficiencia cardiaca es un problema de salud pública de magnitud creciente que genera un gasto de recursos económicos significativo. Su prevalencia ha ido en ascenso debido al gran del número de personas de edad avanzada, el aumento de las expectativas de vida y la mayor supervivencia de enfermos con infarto agudo de miocardio e hipertensos ${ }^{1}$.

Por su parte, la insuficiencia cardiaca genera la activación de mecanismos compensadores entre los cuales el riñón desempeña un papel central ya que este regula la homeostasis hidroelectrolítica y el volumen circulante, y genera un incremento de retención renal de sodio y agua por acción del sistema nervioso simpático (SNS) y el SRAA, lo cual causa disfunción cardiaca y, progresivamente, disfunción renal ${ }^{7}$, que corresponde principalmente a dos enfermedades crónicas: diabetes mellitus e hipertensión arterial; sin embargo, otros factores que inciden hacia falla renal son el envejecimiento poblacional, el tabaquismo, la hiperlipidemia y los antecedentes familiares de enfermedad renal crónica ${ }^{11,12}$.

Esta enfermedad, al igual que la insuficiencia cardiaca, se ha convertido en un problema de salud pública ya que según la información entregada por el tercer reporte de la National Health and Nutrition Examination Survey se estima que cerca de 6,2 millones de individuos mayores de 12 años tienen compromiso de la función renal ${ }^{1}$. Cualquier grado de disfunción renal aun leve, puede aumentar el riesgo cardiovascular y asociarse a mayor mortalidad en forma independiente a otros factores de riesgo ${ }^{7}$. Según Gnanaraj y Radhakrishnan, en su publicación "Cardio-renal syndrome" la incidencia del síndrome cardiorrenal depende del tipo de síndrome que se presente; no obstante, se debe saber que la insuficiencia renal aguda ocurre entre el 25 al 33\% de la falla cardiaca aguda descompensada.

\section{Etiología}

El síndrome cardiorrenal se caracteriza por un alto grado de interacciones entre el corazón y el riñón ${ }^{8}$, pero, poco 
Tabla 1 Clasificación del síndrome cardiorrenal

Síndrome cardiorrenal (SCR) definición: desorden fisiopatológico que afecta al corazón y riñón, y en el que la disfunción de un órgano induce a la disfunción del otro, ya sea de forma crónica o aguda.

SCR tipo 1 (síndrome cardiorrenal agudo): rápido compromiso de la función cardiaca (shock cardiogénico, insuficiencia cardiaca o descompensada) que genera una lesión renal aguda.

Biomarcadores ET-1, troponina, CPK-MB.

SCR tipo 2 (síndrome cardiorrenal crónico): anormalidad crónica de la función cardiaca (insuficiencia cardiaca congestiva crónica) que potencialmente causa en forma progresiva enfermedad o insuficiencia renal crónica. Biomarcadores ET-1, BNP. SCR tipo 3 (síndrome renocardiaco agudo): rápido compromiso de la función renal (isquemia renal aguda, necrosis tisular o glomerulonefritis aguda) que causa alteración cardiaca (insuficiencia cardiaca, arritmias, isquemia). Biomarcadores TNF-alfa, IL-1, IL-6, IL-8.

SCR tipo 4 (síndrome renocardiaco crónico): enfermedad renal crónica (enfermedad glomerular o intersticial crónica) que contribuye al deterioro de la función cardiaca, a hipertrofia ventricular y/o eleva el riesgo de un evento cardiovascular adverso. Biomarcadores PTH, CPP, cystatin C.

SCR tipo 5 (síndrome cardiorrenal secundario): condición sistemática (diabetes mellitus, sepsis, amiloidosis) que causa disfunción cardiaca y renal.

ET-1: endotelina-1; CPK-MB: creatinina fosfoquinasa-MB; BNP: péptido natriurético tipo B; TNF: factor de necrosis tumoral; IL: interleuquina; PTH: hormona paratiroides; CPP: fosfato de calcio.

se sabe de los mecanismos intrínsecos de la comunicación cardiorrenal ${ }^{3}$, para lo cual la interacción mutua de ambos órganos en la regulación hemodinámica y el conocimiento del desarrollo de otros mecanismos confieren gran complejidad $^{10}$, ya que puede tratarse de una asociación por la coexistencia de factores de riesgo cardiovascular con la enfermedad renal crónica ${ }^{3}$. Cruz y Bagshaw describen detalladamente los aspectos epidemiológicos y clínicos de los distintos tipos de síndrome cardiorrenal $^{2}$, que integran la clasificación de Ronco basada en la fisiopatología, el periodo de tiempo y la naturaleza de la patología cardiaca y renal ${ }^{6}$; la cual fue adoptada por el grupo del consenso Acute Dialysis Quality Initiative (ADQI) ${ }^{2}$. Recientemente, Hatamizadeh et al., hicieron una propuesta basada en las manifestaciones clínicas más importantes ${ }^{10}$ (tabla 1 ), de acuerdo con la cual el síndrome cardiorrenal se clasifica en cinco subtipos 1 , según se trate de una lesión aguda o crónica y si el compromiso de la lesión es evidentemente simultáneo ${ }^{12}$ (fig. 1).

\section{Tipo 1 o síndrome cardiorrenal agudo}

Consiste en un compromiso rápido de la función cardiaca ${ }^{8}$ que genera una lesión renal aguda ${ }^{12}$; puede dividirse en cuatro subgrupos clínicos: edema pulmonar hipertensivo con



Figura 1 Clasificación del síndrome cardiorrenal. 
función ventricular izquierda preservada ${ }^{13}$, falla cardiaca descompensada, insuficiencia ventricular derecha y choque cardiogénico $^{10}$.

La importancia clínica de cada mecanismo puede variar de paciente a paciente y de una situación a otra ${ }^{13}$. El principio clínico básico es la lesión renal aguda por hipoperfusión, perpetuada por la asociación de bajo gasto cardiaco, además del aumento marcado en la presión venosa, que conduce a congestión renal ${ }^{10}$.

\section{Tipo 2 o síndrome cardiorrenal crónico}

Refleja el deterioro crónico de la función cardiaca, que causa insuficiencia renal crónica progresiva ${ }^{1}$, manifestándose inicialmente con anormalidades cardiovasculares crónicas, como la insuficiencia cardiaca crónica, la fibrilación auricular, la miocardiopatía o la cardiopatía isquémica². Este síndrome es común y se ha informado en el $63 \%$ de los pacientes hospitalizados con insuficiencia cardiaca congestiva ${ }^{8}$.

\section{Tipo 3 o síndrome renocardíaco agudo}

Constituye deterioro agudo de la función renal que lleva a disfunción cardiaca ${ }^{13}$. Los mecanismos de lesión son múltiples, dados por sobrecarga de líquidos que contribuyen al desarrollo de edema pulmonar ${ }^{10}$, alteraciones del metabolismo mineral en las concentraciones de calcio y fósforo ${ }^{2}$, hiperkalemia (genera trastornos del ritmo y la conducción cardiaca), uremia (afecta directamente la contractilidad miocárdica) y finalmente isquemia renal, que puede inducir a inflamación y apoptosis cardiaca ${ }^{10}$.

\section{Tipo 4 o síndrome renocardíaco crónico}

Se caracteriza por afectación cardiovascular en pacientes con enfermedad renal crónica ${ }^{2}$. Trae como consecuencia hipertrofia ventricular, remodelamiento y favorecimiento de eventos cardiovasculares tales como infarto agudo de miocardio o enfermedad cerebro vascular (ECV) ${ }^{1}$.

\section{Tipo 5 o síndrome cardiorrenal secundario}

Se caracteriza por la presencia simultánea de disfunción renal y cardiaca, asociada a trastornos sistémicos agudos o crónicos ${ }^{10}$. Dentro de los agudos están el choque séptico y el hemorrágico de origen traumático o las quemaduras múltiples ${ }^{2}$, el lupus eritematoso sistémico, la diabetes mellitus, la malaria, la vasculitis, la cirrosis hepática, entre otros $^{8}$. En la fase aguda, la sepsis severa representa la condición más común y grave que puede afectar a los órganos e inducir lesión renal aguda, mientras que lleva a una profunda depresión miocárdica ${ }^{13}$.

\section{Fisiopatología}

Marin et al., ${ }^{14}$ mencionan la estrecha relación existente entre las enfermedades cardiovasculares y la enfermedad renal (tabla 2), en tanto que Yoshitsugu et al. en su obra "Currentand Potential Therapeutic Strategies for Hemodynamic Cardiorrenal Syndrome" refiere que estos dos órganos se relacionan y dan origen al síndrome cardiorrenal mediante factores ateroscleróticos y hemodinámicos, uremia, tromboembolia, disfunción endotelial, desorden electrolítico, autonómico y metabólico, acidosis metabólica, anemia, disbalance protéico y factores genéticos.

Tabla 2 Relación entre insuficiencia cardiaca e insuficiencia renal crónica

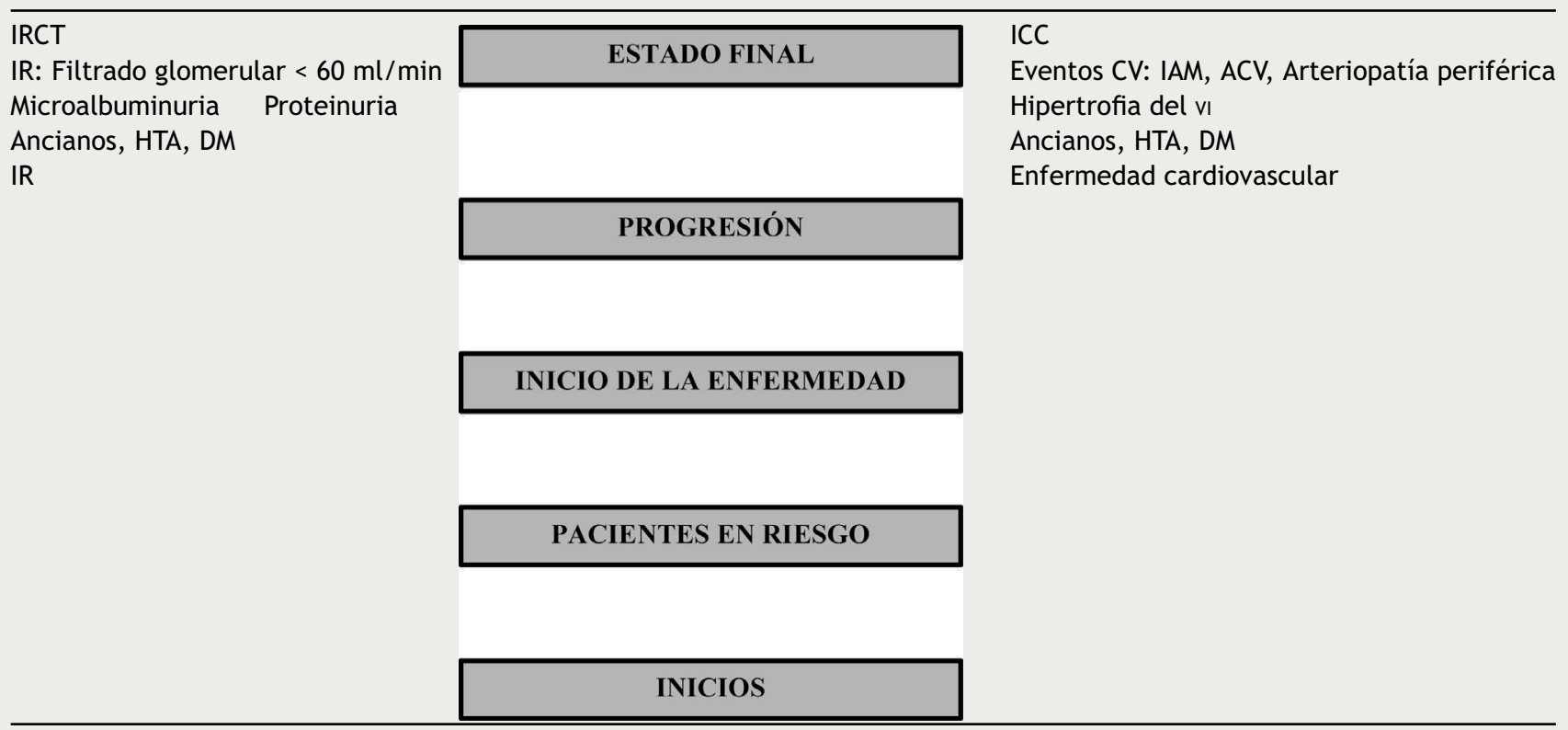

IR: insuficiencia renal. HTA: hipertensión arterial. DM: diabetes mellitus. IRCT: insuficiencia renal crónica terminal. vI: ventrículo izquierdo. CV: cardiovascular. IAM: infarto agudo de miocardio. ACV: ataque cerebrovascular. ICC: insuficiencia cardiaca crónica. 


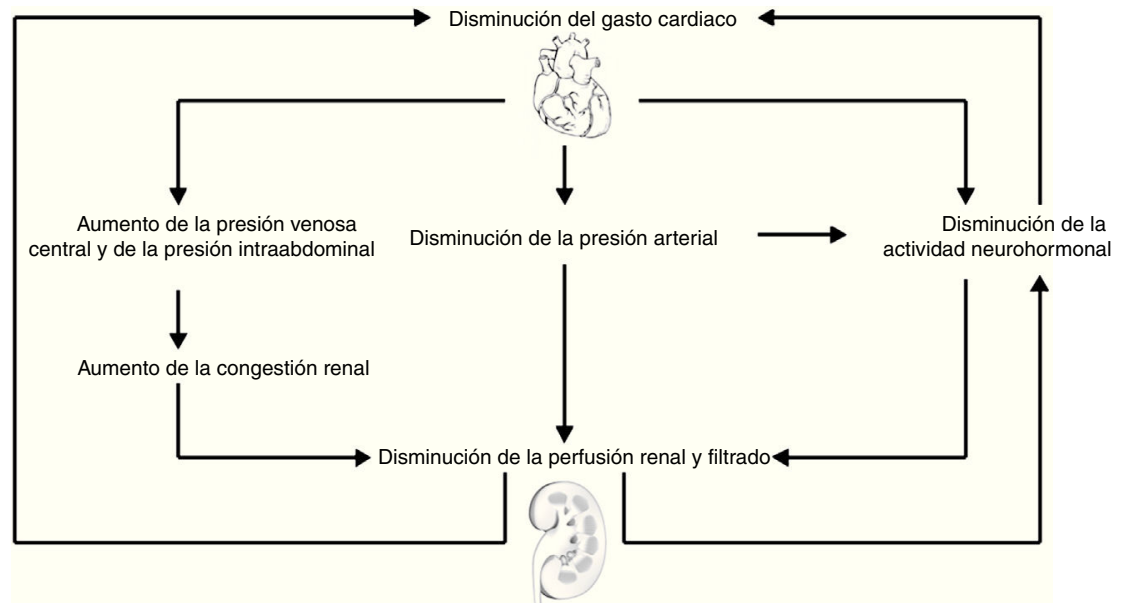

Figura 2 Efecto de la insuficiencia cardiaca en la función renal.

Es claro que la disfunción cardíaca asociada al empeoramiento hemodinámico ocasiona la activación de mecanismos compensadores en los órganos sistémicos; en cuyo caso el riñón ejerce un papel central ya que regula la homeostasis de electrolitos y volumen ${ }^{15}$; por tanto, la disfunción cardiaca aguda conllevará daño renal agudo (síndrome cardiorrenal agudo) ${ }^{16-19}$, cuyos mecanismos son múltiples y complejos ${ }^{20}$ (fig. 2). Al mismo tiempo, los pacientes con disminución en la fracción de eyección del ventrículo izquierdo presentan manifestaciones más severas frente a los pacientes que tienen una fracción de eyección preservada, como también una incidencia mayor al $70 \%$ en pacientes con choque cardiogenico ${ }^{21}$. Basándose en lo anterior, la causa principal e inicial para la falla renal aguda es la hipoperfusión por disminución del gasto cardiaco con marcado incremento en la presión venosa, que ocasiona congestión renal ${ }^{19}$. La segunda causa importante es la disminución de la capacidad de respuesta diurética, por consecuencia de los fenómenos fisiológicos de frenado diurético (retención de sodio post-diuresis) ${ }^{22}$, así como también la afectación por activación simpática, hormonal y neurohumoral en su intento de compensar la disminución del gasto cardiaco $^{19}$ (fig. 3). La retención de sodio y agua por parte del riñón, puede deteriorar aun más la función cardíaca por incremento de la

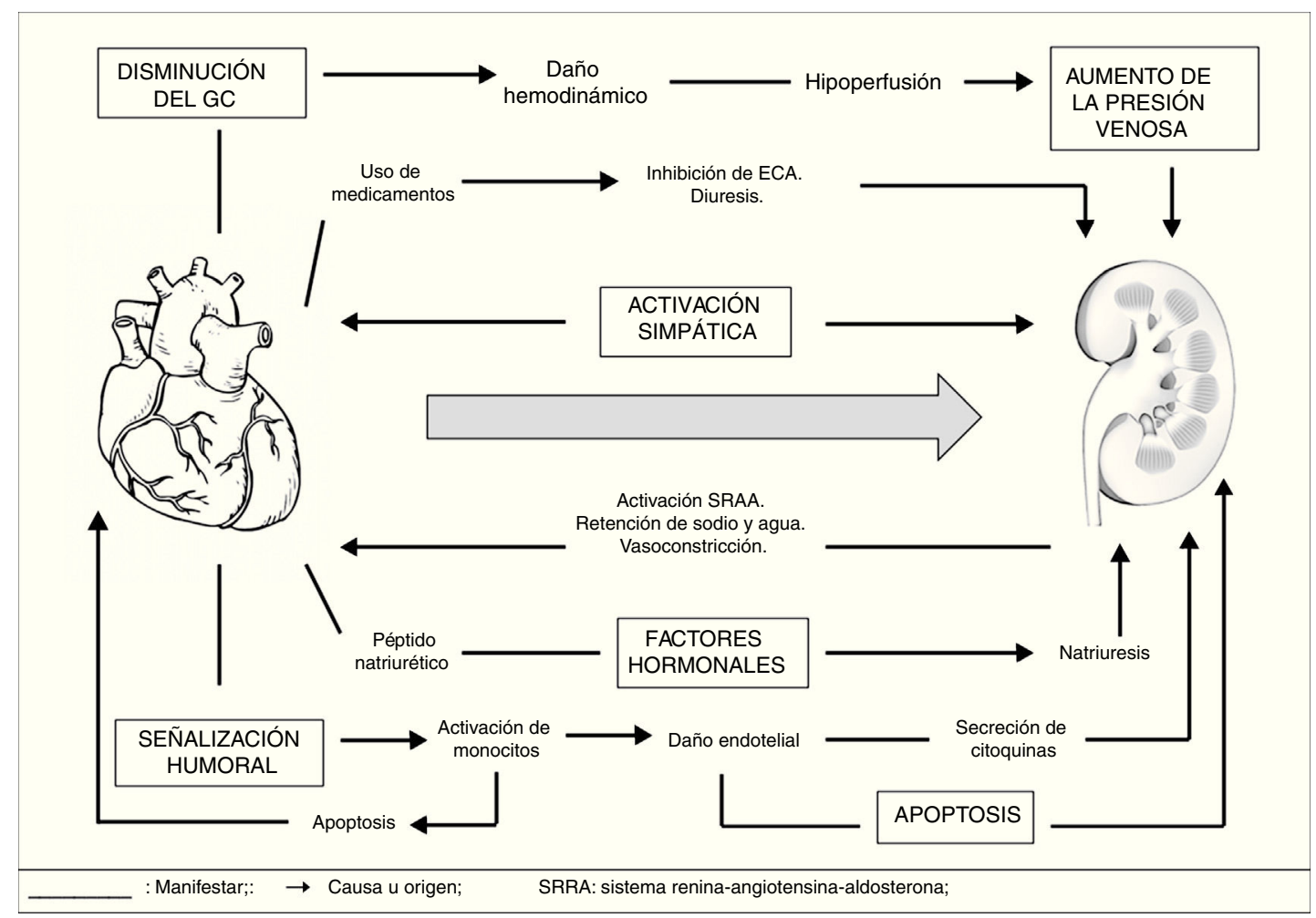

Figura 3 Síndrome cardiorrenal tipo I. 
Tabla 3 Biomarcadores cardiorrenales

\begin{tabular}{|c|c|}
\hline Biomarcadores & Asociado a lesiones \\
\hline N-GAL (Lipocalina) & Isquemia y nefrotoxinas. \\
\hline $\begin{array}{l}\text { Citoquinas (IL-6, IL-8, } \\
\text { IL-18, TNF) }\end{array}$ & Tóxicos, lesión, apoptosis. \\
\hline Cistatina C & $\begin{array}{l}\text { Estimación de la función } \\
\text { renal. Lesión túbulo } \\
\text { proximal. }\end{array}$ \\
\hline KIM-1 & Isquemia y nefrotoxinas. \\
\hline NHE3 & $\begin{array}{l}\text { Isquemia pre-renal, } \\
\text { post-renal de FRA. }\end{array}$ \\
\hline GST & Lesión proximal y distal. \\
\hline L-FABP & Isquemia y nefrotoxinas. \\
\hline Netrin-1 & $\begin{array}{l}\text { Isquemia, nefrotoxinas y } \\
\text { sepsis. }\end{array}$ \\
\hline Creatinina & $\begin{array}{l}\text { Daño en el riñón, infección o } \\
\text { reducción del flujo de sangre }\end{array}$ \\
\hline Troponina & Isquemia miocárdica. \\
\hline Mioglobina & Isquemia, inflamación. \\
\hline Mieloperoxidasa & $\begin{array}{l}\text { Metabolismo de los miocitos, } \\
\text { inflamación, estrés oxidativo. }\end{array}$ \\
\hline Péptido natriurético tipo $B$ & $\begin{array}{l}\text { Falla cardiaca } \\
\text { descompensada. }\end{array}$ \\
\hline
\end{tabular}

IL: interlukinas; KIM: kidney injury molecule; GST: glutathione Stransferase; NHE: sodium- hydrogen exchanger; L-FABP: L-type fatty acid binding protein; FRA: falla renal aguda.

precarga y de la postcarga, dando lugar a un círculo vicioso que origina el trabajo en equipo entre la disfunción renal y cardíaca para amplificar la progresión del fallo de cada órgano de forma individual ${ }^{15,23}$.

En la fisiopatología de este proceso, intervienen en forma compleja el SRAA, el balance óxido nítrico/especies reactivas de oxígeno (ROS), la inflamación y el sistema nervioso simpático; todas estas variables estarían interrelacionadas y serían responsables de la ateroesclerosis acelerada, la remodelación, la hipertrofia ventricular izquierda y la progresión de la enfermedad renal ${ }^{24}$. El síndrome cardiorrenal tipo I, tiene una progresión rápida al igual que sus manifestaciones a través de los biomarcadores en la falla renal aguda. Uno de los biomarcadores más rápidos para su detección en la sangre o en la orina, es la lipocalina asociada con la gelatinasa de neutrófilos (NGAL) ${ }^{25-28}$, además de la creatinina sérica ${ }^{29,30}$, la cistatina $C$ y la molécula de lesión renal $(\mathrm{KIM}-1)^{19}$ entre otros (tabla 3$)$.

El síndrome cardiorrenal tipo II (síndrome cardiorrenal crónico), se caracteriza por anormalidades crónicas en la función cardiaca, por ejemplo insuficiencia cardiaca congestiva crónica que causa enfermedad renal crónica (ERC) ${ }^{29}$; es importante saber, que el síndrome cardiorrenal tipo II se manifiesta principalmente con una disminución del gasto cardiaco, disfunción endotelial acompañada de hipertensión que con lleva disfunción renal ${ }^{19}$, al igual que el uso de medicamentos para falla cardiaca que pueden empeorar la función renal y la hipovolemia asociada a la diuresis ${ }^{20}$.

En la actualidad, el déficit de eritropoyetina se relaciona con anemia marcada, hipoxia, activación simpática y del SRAA e hipertrofia ventricular izquierda en este tipo de síndrome cardiorrenal $^{30}$; de la misma manera, que se relaciona con las anormalidades neurohormonales que producen mediadores vasoconstrictores (angiotensina, epinefrina, endotelina) y factores vasodilatadores (natriurético óxido nítrico) como mecanismos compensadores, que finalmente harán que el síndrome perjudique por hipoperfusión renal crónica, apoptosis y fibrosis (fig. 4) ${ }^{19}$.

El síndrome cardiorrenal tipo III (síndrome renocardiaco agudo) se caracteriza por afectación de la función renal (insuficiencia renal aguda, isquemia, glomerulonefritis) conlleva disfunción cardiaca aguda (falla cardiaca, arritmias, isquemia $)^{31}$, ya que la lesión renal aguda puede afectar de muchas formas la función cardiaca. De aquí que la retención de líquido contribuye al desarrollo de edema pulmonar, como la hipercalemia a arritmias y paro cardiaco $^{19}$. La uremia no tratada afecta la contractilidad miocárdica mediante los factores depresores miocárdicos ${ }^{32,33}$. La acidemia produce un efecto inotrópico negativo ${ }^{34}$, que podría originar un disbalance electrolítico y dar origen a un incremento en el riesgo de arritmias $^{35}$; del mismo modo, dicha acidemia produce una vasoconstricción pulmonar, lo que significa que contribuye a una falla cardiaca derecha ${ }^{36}$. Finalmente, la isquemia renal podría originar inflamación y apoptosis cardiaca $^{37}$ (fig. 5). De manera que la sensibilidad y especificidad de los biomarcadores cardiacos, pueden ayudar al diagnóstico y tratamiento, a sabiendas que cuanto más temprano, mayor efectividad, al igual que los biomarcadores renales en el síndrome cardiorrenal tipo $\mathrm{I}^{38}$. Los biomarcadores cardiacos más significativos e importantes son la troponina ${ }^{39,40}$, el péptido natriurético tipo $B(B N P)^{41}$, la mieloperoxidasa ${ }^{42}$ y citoquinas como el factor de necrosis tumoral, IL-1, IL-6 ${ }^{43,44}$ (tabla 3).

El síndrome cardiorrenal tipo IV (síndrome renocardiaco crónico) se caracteriza por una condición de enfermedad renal crónica que conduce a disminución de la función cardiaca, hipertrofia ventricular, disfunción diastólica y/o aumento del riesgo para evento cardiovascular ${ }^{45}$, debido principalmente a alteraciones renales como anemia, inflamación crónica, producción de citoquinas y retención de sodio y agua $^{19}$ (fig. 6). En última instancia, el síndrome cardiorrenal tipo $\mathrm{V}$ (síndrome cardiorrenal secundario) se caracteriza por la presencia combinada de disfunción cardiaca y renal, asociada a desórdenes sistémicos agudos o crónicos $^{46}$; tales como enfermedad sistémica, diabetes, amiloidosis, vasculitis y sepsis. Dichas alteraciones producen activación del sistema simpático, estrés neurohormonal, inflamación, hipoperfusión, aumento de las presiones, isquemia, hipoxia entre otras ${ }^{47}$ (fig. 7).

\section{Diagnóstico}

Se basa tanto en los datos de laboratorio como en los métodos diagnósticos de imagen ${ }^{48}$ para la identificación temprana de disfunción renal, ya que es esencial para el tratamiento de síndrome cardiorrenal $^{9}$, los criterios propuestos para el diagnóstico de síndrome cardiorrenal son: insuficiencia cardiaca, insuficiencia renal y compromiso hemodinámico (tabla 4$)^{10}$. El estándar de oro para la medición de la función renal parece ser la determinación de la tasa de filtración glomerular (TFG) (ya sea por métodos directos o mediante estimaciones basadas por biomarcadores) $)^{49}$ como la creatinina sérica como indicador de daño renal $^{9}$, la lipocalina asociada con la 


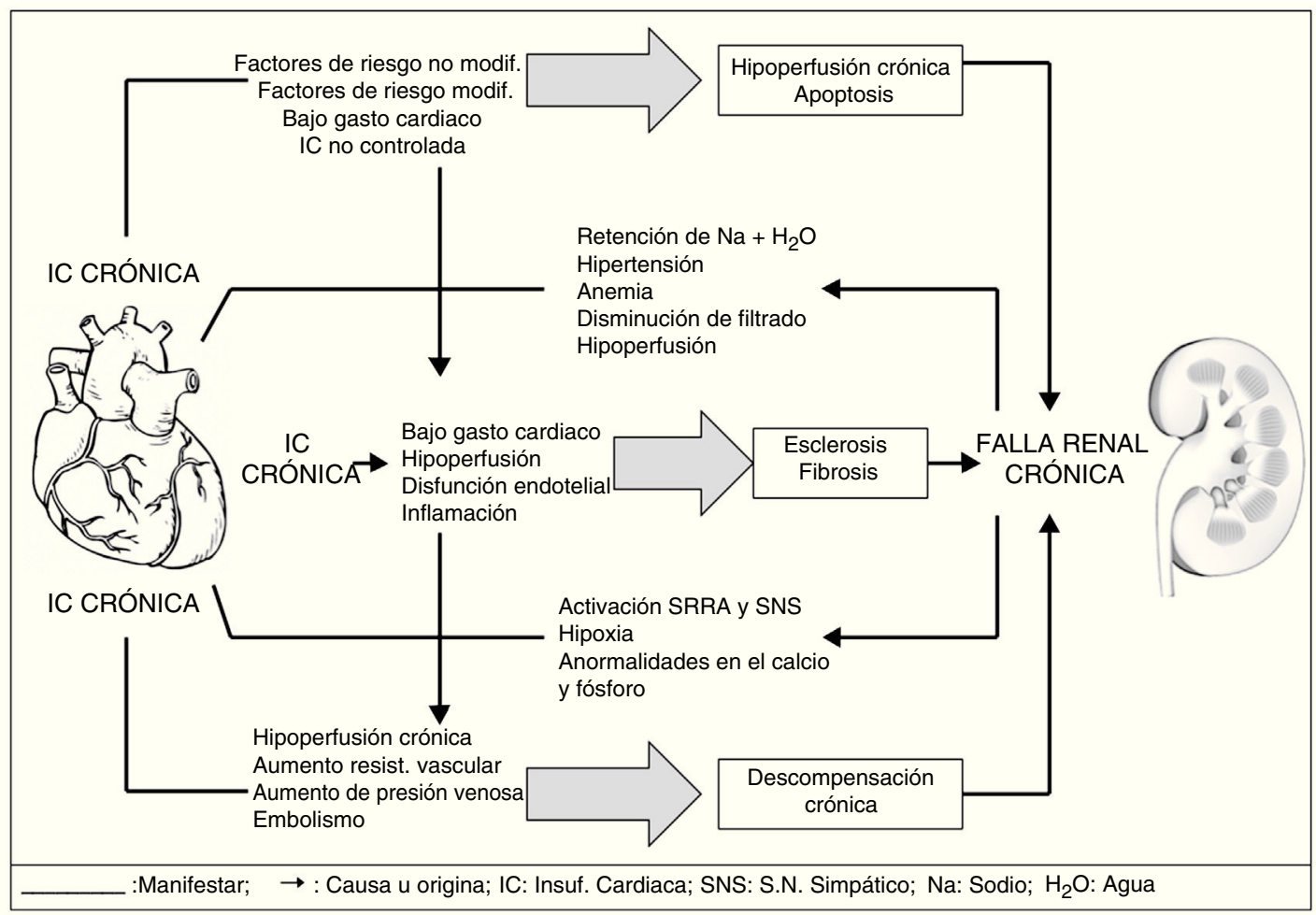

Figura 4 Síndrome cardiorrenal tipo II.

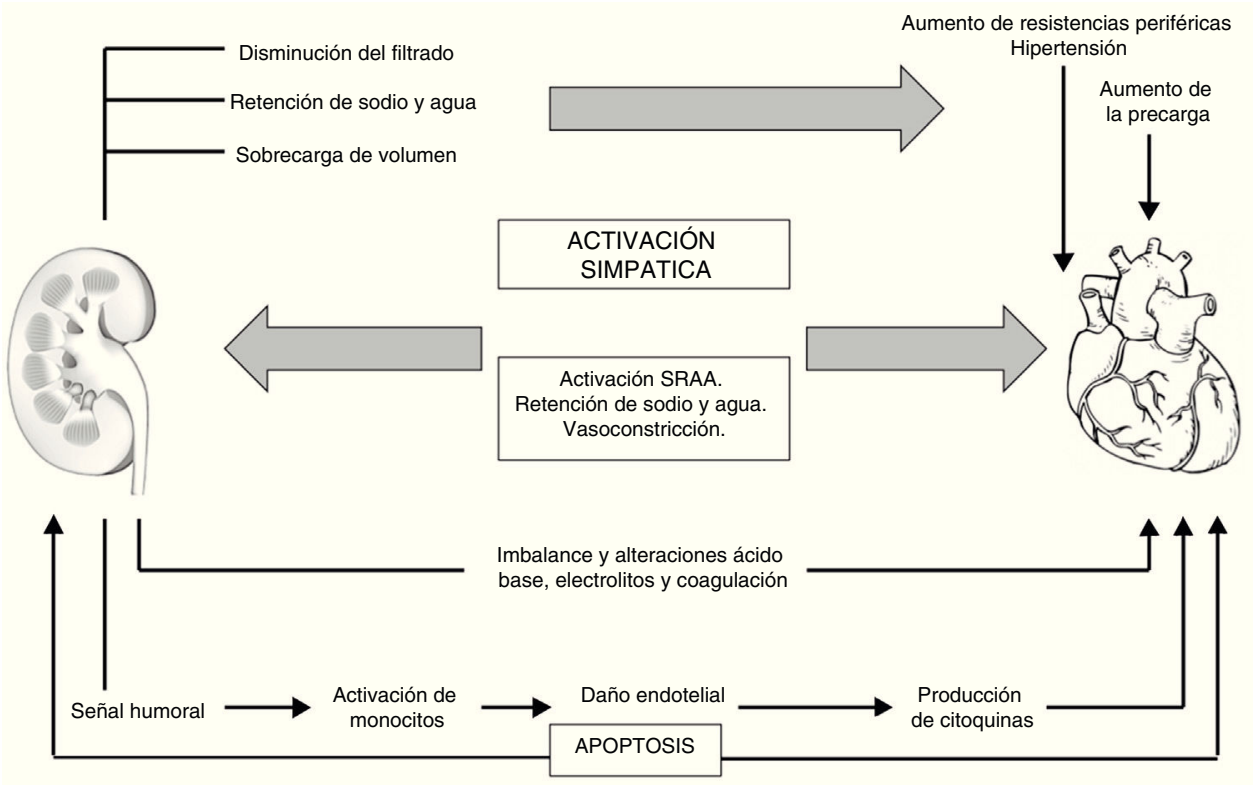

Figura 5 Síndrome cardiorrenal III.

gelatinasa de neutrófilos (NGAL) como marcador para identificar la lesión renal aguda e indicador de falla cardiaca ${ }^{10}$, la hiponatremia, que indica un exceso de hormona antidiurética lo cual es marcador de daño renal $^{9}$ y por último la monitorización hemodinámica que puede auxiliar al diagnóstico oportuno. Los pacientes hospitalizados por insuficiencia cardiaca aguda deben ser monitorizados desde el punto de vista hemodinámico y seguidos con biomarcadores fisioló- gicos para la detección temprana de lesión o daño renal. En forma paralela, el péptido natriurético tipo $B$ (BNP), como el péptido natriurético cerebral N-terminal (NT-pro BNP) son utilizados como biomarcadores para insuficiencia cardiaca descompensada y sobrecarga de volumen ${ }^{10}$. Otros cambios pueden ser causados por la presencia de insuficiencia cardíaca y su tratamiento con diuréticos, dentro de los que se destacan aldosteronismo secundario, 


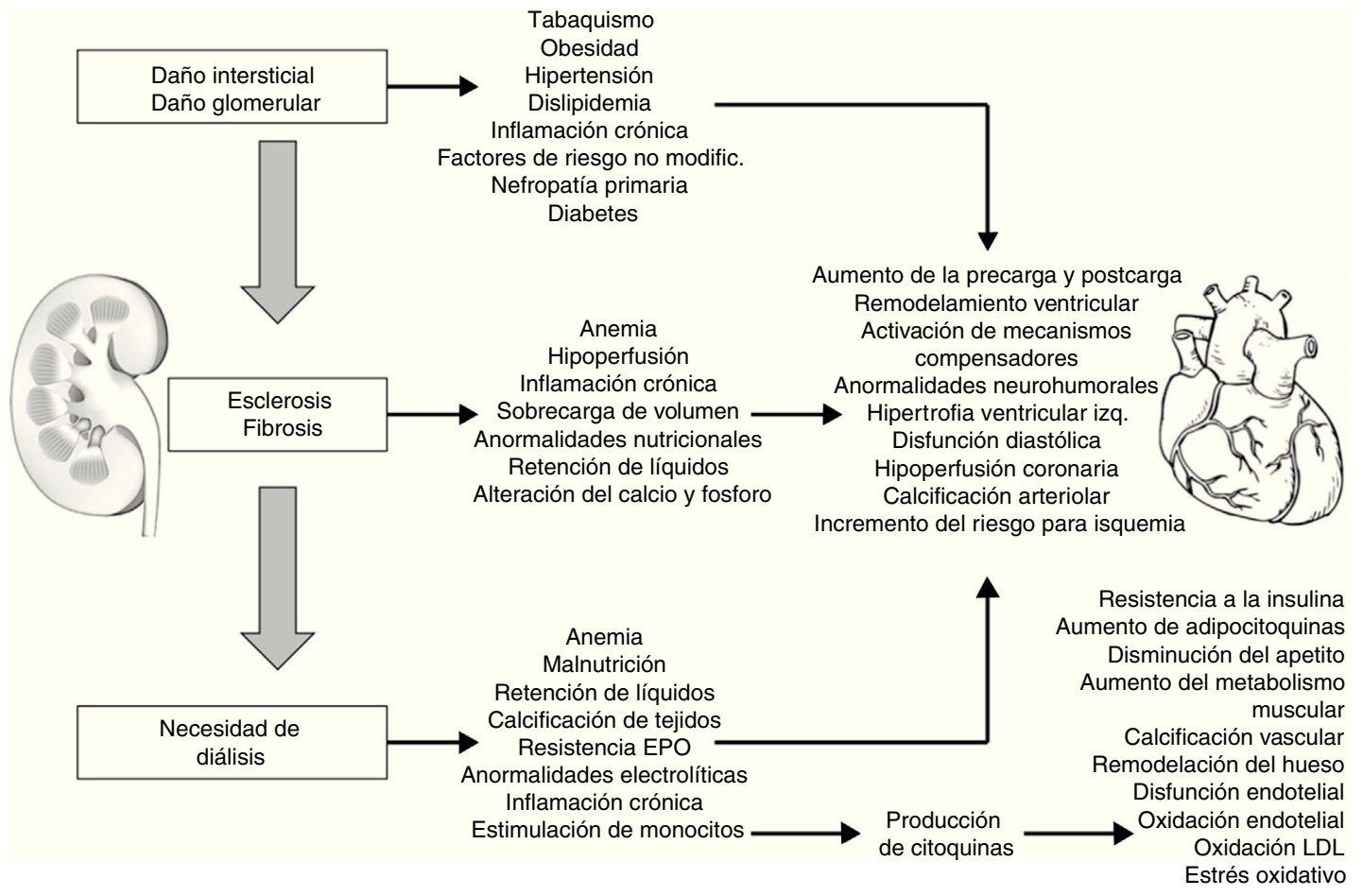

Figura 6 Síndrome cardiorrenal IV.



Figura 7 Síndrome cardiorrenal v.

deficiencia de micronutrientes y alteraciones hidroelectrolíticas que contribuyen al deterioro clínico progresivo ${ }^{51}$. Así mismo, los niveles elevados de electrolitos séricos como el potasio y el calcio predisponen al desarrollo de arritmias, mientras que los niveles de cistatina $C$ pueden ser marcadores de insuficiencia renal aguda ${ }^{10}$. Además, resulta importante brindar un espacio a la cistatina $C$ ya que, a diferencia de la creatinina y el nitrógeno ureico en sangre, ésta es independiente de la masa muscular, el consumo de proteínas o el catabolismo, y de ahí que diferentes autores sugieran su utilización como marcador más específico y exacto del filtrado glomerular ${ }^{52}$. Ahora bien, Constantin et al. ${ }^{52}$ en el 2016 confirmaron que la cistatina $C$ muestra alteraciones de la tasa del filtrado glomerular más rápido y preciso que sus antecesores, lo que la convierte en un marcador precoz de falla renal en pacientes con insuficiencia cardíaca aguda ${ }^{53}$. Otro aspecto importante es la evaluación ecográfica renal, la cual se convierte en una parte 
Tabla 4 Criterios diagnósticos

\begin{tabular}{lll}
\hline Insuficiencia cardiaca y renal aguda tipo 1 y 3 & Insuficiencia cardiaca y renal crónica tipo 2 y 4 & Enfermedades sistémicas tipo 5 \\
\hline Insuficiencia cardiaca aguda & Insuficiencia cardiaca crónica & $\begin{array}{c}\text { Afección sistémica } \\
\text { Hipertermia }\end{array}$ \\
Vértigo & Hipotermia \\
Síncope & Ortopnea & Fiebre y escalofrío \\
Dolor torácico & Disnea de esfuerzo & Taquicardia \\
Taquicardia & Disnea paroxística nocturna & Alteración del pulso \\
Taquipnea y disnea & Tos no productiva crónica & Taquipnea \\
Arritmia & Edema & Confusión \\
Ausencia de murmullo vesicular & Ritmo de galope & Vértigo \\
Hipertensión & Aumento de índice cardiaco & \\
& Elevación de presión venosa & \\
Insuficiencia renal aguda & Hepatomegalia & \\
Fiebre y malestar general & Insuficiencia renal crónica & \\
Deshidratación & Palidez generalizada & \\
Edema & Alteración del estado mental & \\
Taquicardia & Anorexia, náuseas y vómito & \\
Hipotensión ortostática & Edema & \\
Dolor en flanco & Parestesias & \\
Disminución del volumen urinario & Frote pericárdico & \\
& Estertores pulmonares & \\
\hline
\end{tabular}

fundamental para el diagnóstico de obstrucción renal ${ }^{50}$ ya que permite observar el tamaño del parénquima renal y así determinar si es normal o no a fin de examinar la relación cortico-medular ${ }^{54}$. Además, la evaluación Doppler permite documentar la normalidad del flujo sanguíneo intraparenquimatoso a nivel de las arterias interlobulares ${ }^{54}$ y así poder cuantificar la resistencia vascular renal. En la radiografía de tórax se puede evidenciar falla cardiaca con cardiomegalia, líneas B de Kerley y fluido alveolar ${ }^{10}$, así como también hipertrofia ventricular, estenosis e insuficiencia valvular, derrame pericárdico y aneurisma de la aorta ascendente o descendente ${ }^{54}$.

\section{Tratamiento}

El síndrome cardiorrenal habitualmente es secundario a múltiples factores que actúan de manera simultánea. Estos incluyen las alteraciones hemodinámicas que se acompañan de elevación de las presiones de llenado del corazón y congestión venosa renal ${ }^{54}$, lo que produce una disminución del gradiente de presión arterio-venoso del riñón, así como de la presión arterial media y de perfusión renal, lo que conduce a reducir la filtración glomerular ${ }^{8}$. Todos los pacientes con síndrome cardiorrenal necesitan un manejo multidisciplinario con cardiólogo y nefrólogo, así como una vigilancia estrecha tanto clínica como de laboratorio ${ }^{5}$. El tratamiento incluye el uso prudente y combinado de fármacos o intervenciones apropiadas para aliviar síntomas de isquemia, función hemodinámica y estado circulatorio del paciente ${ }^{55}$.

Cuando el órgano primario que se afecta es el corazón, se debe instaurar tratamiento para síndrome cardiorrenal tipo 1 con diuréticos, inotrópicos y asistencia ventricular ${ }^{55,56}$.
Ahora bien, es de suma importancia reconocer cuando hay sobrecarga de volumen o en el peor de los casos resistencia a los diuréticos; para ello, es preciso conocer signos y síntomas clínicos como ortopnea, cardiomegalia, tercer ruido cardiaco (S3), ingurgitación yugular, hepatomegalia y edema $^{56}$. Para el crónico, el tratamiento se basa en inhibidores de la enzima convertidora de angiotensina, antagonistas de receptores angiotensina, antagonistas de aldosterona, estatinas, entre otros ${ }^{57}$. Hasta el momento no existe un tratamiento único y las estrategias están encaminadas a proteger y lograr la mejoría funcional del paciente según sus manifestaciones clínicas ${ }^{10}$ (tabla 5). Cabe concluir con lo referido por el "Consenso colombiano para el diagnóstico y tratamiento de la insuficiencia cardiaca aguda 2014", que los efectos benéficos de los IECA, ARA, antagonistas de la aldosterona y betabloqueadores en insuficiencia cardiaca aguda y síndrome coronario agudo son bien conocidos. Sin embargo, la administración de betabloqueadores en pacientes con síndrome cardiorrenal tipo I se evita hasta que el paciente se haya estabilizado. Los antagonistas de la aldosterona pueden estar asociados con un riesgo pequeño pero hipercalemia significativa; por tanto, es necesaria la monitorización estricta de los electrolitos, en especial en pacientes con enfermedad renal crónica, diabéticos, ancianos y uso concomitante de medicamentos retenedores de potasio. La nitroglicerina, el dinitrato de isosorbide, el nitropusiato y la hidralazina han sido usados en síndrome cardiorrenal cuando hay contraindicación para el empleo de IECA o ARA. De otro lado, los antagonistas de la adenosina y los antagonistas de la vasopresina no demostraron resultados clínicos benéficos, razón por la cual no están recomendados. Debe evaluarse la anemia y la cinética del hierro, ya que con frecuencia se asocian con el síndrome cardiorrenal (Sociedad 
Tabla 5 Estrategias terapéuticas en el manejo del síndrome cardiorrenal

Potenciales blancos terapéuticos en el manejo del síndrome cardiorrenal

\begin{tabular}{ll}
\hline Blanco terapéutico & Estrategia terapéutica \\
Alteraciones & $\rightarrow$ Antagonistas de receptor V2 de \\
hemodinámicas & vasopresina \\
& $\rightarrow$ Bloqueadores del receptor de \\
& aldosterona \\
& $\rightarrow$ Sensibilizadores de calcio \\
& $\rightarrow$ Antagonistas del receptor de \\
& endotelina \\
& $\rightarrow$ Activadores de miosina cardiaca \\
Uremia & $\rightarrow$ Hemodiálisis de alto flujo \\
Arterioesclerosis, & $\rightarrow$ Inhibidores de la enzima \\
disfunción & convertidora de angiotensina \\
endotelial y & $\rightarrow$ Bloqueadores del receptor de \\
tromboembolia & aldosterona \\
& $\rightarrow$ Antagonistas del receptor \\
& endotelina óxido nítrico \\
Neurohumorales & $\rightarrow$ Bloqueadores del receptor de \\
& aldosterona \\
& $\rightarrow$ Antagonistas del receptor A1 \\
& $\rightarrow$ Inhibidores de renina \\
& $\rightarrow$ Soporte nutricional (antioxidantes, \\
ácidos grasos) \\
Anemia y \\
alteraciones del \\
hierro
\end{tabular}

Colombiana de Cardiología 2014). Por otra parte, para el síndrome cardiorrenal tipo 3, el manejo debe ser direccionado a las manifestaciones y causas de la insuficiencia renal aguda. En el síndrome cardiorrenal tipo 4 se requiere un enfoque multifactorial para la falla cardiaca secundaria a la insuficiencia renal crónica. Por último, según Hadjiphilippou y Sui Phin Kon ${ }^{58}$ en su más reciente publicación "Cardiorrenal syndrome: review of our current understanding" el tratamiento ideal para el síndrome cardiorrenal tipo 5 se divide en tres categorías:

1. Mejorar la función cardiaca (inotrópicos, vasodilatadores, resincronización cardiaca, betabloqueadores y receptores antagonistas de adenosina).

2. Disminuir la sobrecarga de volumen (diuréticos, receptores antagonistas de vasopresina y ultrafiltración).

3. Drogas para reducir la fracción de eyección y la enfermedad renal crónica (antagonistas del sistema renina-angiotensina-aldosterona).

\section{Prevención}

Ya se comentó previamente la relación del daño cardiovascular y la enfermedad renal crónica; aproximadamente el 30\% de los pacientes con insuficiencia cardiaca tienen enfermedad renal crónica, lo cual se asocia con peor supervivencia y mayor hospitalización ${ }^{3}$, debido a esto deben implementarse medidas preventivas para garantizar un óptimo estado de completo bienestar físico, mental y social; para ello, el manejo del riesgo cardiovascular en personas con factores de riesgo debe ser integral ${ }^{3}$, atendiendo todos los aspectos que potencialmente pongan en riesgo al sistema cardiovascular y la función renal ${ }^{11}$; por tanto se deben seguir los siguientes pasos:

1. Establecer la situación de cada persona con relación a los factores de riesgo modificables conocidos como tabaquismo, sedentarismo, obesidad, HTA, dislipidemia, síndrome metabólico ${ }^{3}$.

2. Informar al paciente los factores de riesgo que presenta y las medidas que deben tomarse en la búsqueda su implicación para el control de los mismos:

- Toma regular de la tensión arterial.

- Dieta reducida en contenido proteico, bajo contenido en sodio y baja en grasas de contenido animal.

- Ejercicio físico dirigido.

- Seguimiento periódico adecuado en consulta externa con estudios de control.

3. Control de glicemia.

4. Control de la dislipidemia.

5. Valoración anual de la función renal.

6. Vigilancia en los niveles de calcio, fósforo y paratohormona.

\section{Responsabilidades éticas}

Protección de personas y animales. Los autores declaran que para esta investigación no se han realizado experimentos en seres humanos ni en animales.

Confidencialidad de los datos. Los autores declaran que han seguido los protocolos de su centro de trabajo sobre la publicación de datos de pacientes.

Derecho a la privacidad y consentimiento informado. Los autores declaran que en este artículo no aparecen datos de pacientes.

\section{Conflicto de intereses}

Los autores declaran no tener ningún conflicto de intereses.

\section{Bibliografía}

1. Echazarreta D. Insuficiencia cardiaca y síndrome cardiorrenal. Rev Insuf Card. 2010;5:92-6.

2. Lobo L, De la serna F. Síndrome cardiorrenal. Rev Fed Arg Cardiol. 2013;2:88-95.

3. Pérez J, Cuevas B. Síndrome cardiorrenal Guías S.E.N. Nefrología. 2008;3:29-32. 
4. Dizeo C, Chirino A, Franchi V, Álvarez S, Forcinito D, Trejo G., et al. Síndrome cardiorrenal como predictor de mala evolución intrahospitalaria en pacientes añosos internados con insuficiencia cardiaca. Insuficiencia cardiaca. 2012;7(3.).

5. Barrio L, Fernández G. Trastorno del agua en el síndrome cardiorrenal. Rev Nefrología. 2011;2:29-34.

6. Suris B, Herrera I, Monteagudo A, Tan I. Síndrome cardiorrenal. AMC. 2014;18(3.).

7. Valle J, Bolaños C. Síndrome cardiorrenal. Revista Medica de Costa Rica Y Centroamerica LXXIII. 2016:151-6.

8. Barros A. Síndrome cardio-renal. Ciencia actual. 2012;2:44-52.

9. Singh NP, Anish Kumar, Taposh, Sarkar. Chapter 132: Cardiorrenal syndrome: an overview. Medicine update. 2013;23:601-4. Revisado el 28-04-17. Disponible online en: www.apiindia. org/medicine_update_2013/chap132.pdf

10. Chávez E, Alemán O, Nando C, Rosas E. Síndrome cardiorrenal: nuevas perspectivas. Rev Mex Cardiol. 2015;26:39-52.

11. Orihuela R, Mendiola R, Gomez J, Galindo E. Síndrome cardiorrenal un doble reto cardiovascular. Rev Sanid Milit Mex. 2014;1:42-7.

12. Llancaqueo M. Síndrome cardiorrenal. Rev Med Clin Condes. 2014:602-12.

13. Ronco C, Mikko H, House A, Nagesh A, Bellomo R. Cardiorrenal syndrome. J Am Coll Cardiol. 2008;52:1527-39.

14. Marín R. Síndrome cardiorrenal. Nefrología. 2004;24 supl 6:17.

15. Lobo L, Álvarez S, De La Serna F. Síndrome cardiorrenal: relación amor/odio. Rev Insuf Cardíaca. 2007;2:62-5.

16. Echazarreta D. Insuficiencia cardiaca y síndrome cardiorrenal. Rev Insuf Card. 2010;1:92-6.

17. Chávez E, Alemán O, Nando C, Rosas E. Síndrome cardiorrenal: nuevas perspectivas. Rev Mex Cardiol. 2015;26:39-52.

18. Llancaqueo V, Marcel. Síndrome cardiorrenal. Rev Med Clin Condes. 2014:602-12.

19. Jaramillo J, Gomez E, Hernandez E, Saldarriaga C, Flórez N, Buitrago R. Consenso Colombiano para el diagnóstico y tratamiento de la insuficiencia cardiaca aguda. Colombia: Sociedad Colombiana de Cardiologia; 2014.

20. Liang KV, Williams AW, Greene EL, Redfield MM. Acute decompensated heart failure and the cardiorrenal syndrome. Crit Care Med. 2008;36:S75-88.

21. Jose P, Skali H, Anavekar N, Tomson C, Krumholz HM, Rouleau JL, et al. Increase in creatinine and cardiovascular risk in patients with systolic dysfunction after myocardial infarction. J Am Soc Nephrol. 2006;17:2886-91.

22. Yoshitsugu O, Taehee K, Csaba P, Alpesh N, Kamyar K. Current and potential therapeutic strategies for hemodynamic cardiorrenal syndrome. Cardiorrenal Med. 2016;6:83-98, http://dx.doi.org/10.1159/000441283.

23. Gnanaraj J, Radhakrishnan J. Cardio-renal syndrome. F1000 Faculty Rev:2123.

24. Boerrigter G, Burnett JC. Cardiorrenal syndrome in descompensated heart failure: prognostic and therapeutic implications. Current Heart Failure Reports. 2004:113-20. I.

25. Ronco C. NGAL: an emerging biomarker of acute kidney injury. Int J Artif Organs. 2008;31:199-200.

26. Xu S, Venge P. Lipocalins as biochemical markers of disease. Biochim Biophys Acta. 2000;1482:298-307.

27. Mishra J, Ma Q, Prada A, Mitsnefes M, Zahedi K, Yang J, et al. Identification of neutrophil gelatinase-associated lipocalin as a novel early urinary biomarker for ischemic renal injury. J Am Soc Nephrol. 2003;14:2534-43.

28. Supavekin S, Zhang W, Kucherlapati R, Kaskel FJ, Moore LC, Devarajan P. Differential gene expression following early renal ischemia/reperfusion. Kidney Int. 2003;63:1714-24.

29. Herget-Rosenthal S, Marggraf G, Husing J, Goring F, Pietruck F, Janssen $\mathrm{O}$, et al. Early detection of acute renal failure by serum cystatin C. Kidney Int. 2004;66:1115-22.
30. VandeVoorde RG, Katlman TI, Ma Q, et al. Serum NGAL and cystatin $\mathrm{C}$ as predictive biomarkers for acute kidney injury. J Am Soc Nephrol. 2006;17:404A.

31. McCullough PA. Contrast induced nephropathy. J Am Coll Cardiol. 2008;51:1419-28.

32. Jie KE, Verhaar MC, Cramer MJ, van der Putten K, Gaillard CA, Doevendans PA, et al. Erythropoietin and the cardiorrenal syndrome: Cellular mechanisms on the cardiorrenal connectors. Am J Physiol Renal Physiol. 2006;291:F932-44.

33. Uchino S, Bellomo R, Goldsmith D, Bates S, Ronco C. An assessment of the RIFLE criteria for acute renal failure in hospitalized patients. Crit Care Med. 2006;34:1913-7.

34. Blake P, Hasegawa Y, Khosla MC, Fouad-Tarazi F, Sakura N, Paganini EP. Isolation of "myocardial depressant factor(s)" from the ultrafiltrate of heart failure patients with acute renal failure. ASAIO J. 1996;42:M911-5.

35. Meyer TW, Hostetter TH. Uremia. N Engl J Med. 2007;357: 1316-25.

36. Brady JP, Hasbargen JA. A review of the effects of correction of acidosis on nutrition in dialysis patients. Semin Dial. 2000;13:252-5

37. McCullough PA, Sandberg KR. Chronic kidney disease and sudden death: Strategies for prevention. Blood Purif. 2004;22:136-42.

38. Figueras J, Stein L, Diez V, Weil MH, Shubin H. Relationship between pulmonary hemodynamics and arterial $\mathrm{pH}$ and carbon dioxide tension in critically ill patients. Chest. 1976;70:466-72.

39. Berl T, Henrich W. Kidney-heart interactions: epidemiology, pathogenesis, and treatment. Clin J Am Soc Nephrol. 2006;1:8-18.

40. Cameron SJ, Sokoll LJ, Laterza OF, Shah S, Green GB. A multi-marker approach for the prediction of adverse events in patients with acute coronary syndromes. Clin Chim Acta. 2007;376:168-73.

41. Howie-Esquivel J, White M. Biomarkers in acute cardiovascular disease. J Cardiovasc Nurs. 2008;23:124-31.

42. Maisel A, Hollander JE, Guss D, McCullough P, Nowak R, Green G. A multicenter study of B-type natriuretic peptide levels, emergency department decision making, and outcomes in patients presenting with shortness of breath. J Am Coll Cardiol. 2004;44:1328-33.

43. Loria V, Dato I, Graziani F, Biasucci LM. Myeloperoxidase: a new biomarker of inflammation in ischemic heart disease and acute coronary syndromes. Mediators Inflamm. 2008;2008:135625.

44. Krishnagopalan S, Kumar A, Parrillo JE, Kumar A. Myocardial dysfunction in the patient with sepsis. Curr Opin Crit Care. 2002;8:376-88

45. Chen D, Assad-Kottner C, Orrego C, Torre-Amione G. Cytokines and acute heart failure. Crit Care Med. 2008;36:S9-16.

46. Kelly KJ. Distant effects of experimental renal ischemia/reperfusion injury. J Am Soc Nephrol. 2003;14:1549-58.

47. National Kidney Foundation. K/DOQI clinical practice guidelines for chronic kidney disease: Evaluation, classification, and stratification. Am J Kidney Dis. 2002;39:S1-266.

48. Cunningham PN, Dyanov HM, Park P, Wang J, Newell KA, Quigg RJ. Acute renal failure in endotoxemia is caused by TNF acting directly on TNF receptor-1 in kidney. J Immunol. 2002;168:5817-23.

49. Kumar A, Paladugu B, Mensing J, Kumar A, Parrillo JE. Nitric oxide-dependent and independent mechanisms are involved in TNF-alpha-induced depression of cardiac myocyte contractility. Am J Physiol Regul Integr Comp Physiol. 2007;292: R1900-6.

50. Kshatriya S, Kozman H, Siddiqui D, Bhatta L, Liu K, Salah A. El síndrome cardiorrenal en insuficiencia cardíaca: Un paradigma en evolución. Rev Chil Cardiol. 2011;31:160-7.

51. Martins H, Pedro N, Castellano M, Monteiro P, Moura J, Providência $L$. Síndrome cardio-renal os desafíos no tratamento da insuficiência cardíaca. Acta Med Port. 2011;24:285-92. 
52. Núñez J, Miñana G, Santas E, Bertomeu V. Síndrome cardiorrenal en la insuficiencia cardiaca aguda: revisando paradigmas. Rev Esp Cardiol. 2015;68:426-35.

53. Constantin I, Varela C, Del Castillo S, Romeo F, Guzzetti $E$, Citerrio $\mathrm{P}$, et al. Cistatina C como predictor de síndrome cardiorrenal y mal pronóstico en pacientes internados por insuficiencia cardíaca aguda y función renal normal. Rev Argent Cardiol. 2016;84:15-20, http://dx.doi.org/10.7775/ rac. es.v84.i1.7649.

54. Di Lullo L, Fulvio F, De Pascalis A, Marinelli A, Barbera V, Santoboni A. Fisiopatología e diagnosi della sindrome cardio-renale: stato dell'arte e prospettive future. Gital Nefrol. 2013:30.
55. Montenegro A, Silva L, Muñoz M. Síndrome cardio-renal anémico acta médica colombiana, 201;36(3):141-4.

56. Verbrugge F, Mullens W, Tang WH. Management of cardiorenal syndrome and diuretic resistance. Curr Treat Options Cardio Med. 2016;18:11, http://dx.doi.org/10.1007/s11936015-0436-4.

57. Kulkarni M. Cardio renal syndrome. J Nephrol Ther. 2016;6:233, http://dx.doi.org/10.4172/2161-0959.1000233.

58. Hadjiphilippou S, Sui Phin Kon. Cardiorrenal syndrome: review of our current understanding. J R Soc Med. 2016;109:12-7, http://dx.doi.org/10.1177/0141076815616091. 